PROCEEDINGS OF THE

AMERICAN MATHEMATICAL SOCIETY

Volume 136, Number 1, January 2008, Pages 49-53

S 0002-9939(07)08958-7

Article electronically published on September 27, 2007

\title{
GORENSTEIN RINGS AND IRREDUCIBLE PARAMETER IDEALS
}

\author{
THOMAS MARLEY, MARK W. ROGERS, AND HIDETO SAKURAI \\ (Communicated by Bernd Ulrich) \\ Dedicated to Professor Shiro Goto on the occasion of his sixtieth birthday
}

\begin{abstract}
Given a Noetherian local ring $(R, m)$ it is shown that there exists an integer $\ell$ such that $R$ is Gorenstein if and only if some system of parameters contained in $m^{\ell}$ generates an irreducible ideal. We obtain as a corollary that $R$ is Gorenstein if and only if every power of the maximal ideal contains an irreducible parameter ideal.
\end{abstract}

\section{INTRODUCTION}

It is well-known that a commutative Noetherian local ring $(R, m)$ is Gorenstein if and only if $R$ is Cohen-Macaulay and some ideal generated by a system of parameters (s.o.p.) is irreducible. Perhaps less widely known is a result of Northcott and Rees which states that if every ideal generated by an s.o.p. (henceforth called a parameter $i d e a l)$ is irreducible, then $R$ is Cohen-Macaulay [NR, Theorem 1]. Thus, $R$ is Gorenstein if and only if every parameter ideal is irreducible. There are, however, easy examples of non-Gorenstein rings possessing irreducible parameter ideals: (y) $R$ is irreducible in the local ring $R=\mathbb{Q}[[x, y]] /\left(x^{2}, x y\right)$, while $\left(y^{n}\right) R$ is reducible for all $n \geq 2$. In 1982, S. Goto showed that if there exists an s.o.p. $x_{1}, \ldots, x_{d}$ for $R$ such that $\left(x_{1}^{n}, \ldots, x_{d}^{n}\right)$ is irreducible for all sufficiently large $n$, then $R$ is Gorenstein G. Proposition 3.4]. Rephrasing Goto's result, if $R$ is not Gorenstein then for every s.o.p. $x_{1}, \ldots, x_{d}$ of $R$ there exists an integer $n$ (depending on the s.o.p.) such that $\left(x_{1}^{n}, \ldots, x_{d}^{n}\right)$ is reducible. In light of this, given a non-Gorenstein local ring $R$ it is natural to ask whether there exists a uniform exponent $n$ such that $\left(x_{1}^{n}, \ldots, x_{d}^{n}\right)$ is reducible for all s.o.p.'s $x_{1}, \ldots, x_{d}$ of $R$. Our main result (Theorem 2.7) gives an affirmative answer to this question:

Theorem. Let $(R, m)$ be a Noetherian local ring. Then there exists an integer $\ell$ such that $R$ is Gorenstein if and only if some parameter ideal contained in $m^{\ell}$ is irreducible.

Received by the editors August 25, 2006 and, in revised form, September 21, 2006.

2000 Mathematics Subject Classification. Primary 13D45; Secondary 13H10.

Key words and phrases. Gorenstein, system of parameters, irreducible ideal.

The second author was supported for eight weeks during the summer of 2006 through the University of Nebraska-Lincoln's Mentoring through Critical Transition Points grant (DMS-0354281) from the National Science Foundation.

(C)2007 American Mathematical Society Reverts to public domain 28 years from publication 
As a consequence, a local ring $R$ is Gorenstein if and only if every power of the maximal ideal contains an irreducible parameter ideal. The integer $\ell$ identified in this theorem may be taken to be the least integer $\delta$ such that the canonical map

$$
\operatorname{Ext}_{R}^{d}\left(R / m^{\delta}, R\right) \rightarrow \underline{\lim _{\longrightarrow}} \operatorname{Ext}_{R}^{d}\left(R / m^{n}, R\right) \cong H_{m}^{d}(R)
$$

is surjective after applying the functor $\operatorname{Hom}_{R}(R / m,-)$, where $d=\operatorname{dim} R$.

We note that the theorem above has previously been established when $R$ is quasiBuchsbaum or when $R$ has finite local cohomologies and $H_{m}^{i}(R) \neq 0$ for at most two positive values of $i$. These results are either explicitly stated or obtained as easy consequences of [GSa1, Corollary 3.13], GSa2, Theorem 4.2], LR, Corollary 2.2], and $[\mathrm{R}$, Corollary 3.4]

A condition weaker than what is studied here was investigated by Hochster: $R$ is called approximately Gorenstein if every power of $m$ contains an irreducible $m$ primary ideal. While approximately Gorenstein rings must have positive depth, they need not be Cohen-Macaulay. In fact, every complete Noetherian domain is approximately Gorenstein [Ho, Theorem 1.6].

\section{MAIN RESUlts}

As general references for terminology and well-known results, we refer the reader to $\mathrm{Mat}$ or $\mathrm{BH}$. Throughout, $R$ denotes a Noetherian local ring with maximal ideal $m$ and $M$ is a finitely generated $R$-module. The socle of $M$ is defined to be $(0: M m)=\{x \in M \mid m x=0\}$, and is denoted by $\operatorname{Soc}_{R} M$, or $\operatorname{simply} \operatorname{Soc} M$ if there is no confusion about the ring. It is clear that Soc $(-)$ is a left exact covariant functor in a natural way. We identify this functor with $\operatorname{Hom}_{R}(R / m,-)$.

In the sequel we adopt the following notation: For a sequence of elements $\mathbf{x}=$ $x_{1}, \ldots, x_{r}$ of $R$ and $t \in \mathbb{N}$ we let $\mathbf{x}^{t}$ denote the sequence $x_{1}^{t}, \ldots, x_{r}^{t}$. The Koszul chain complex on $R$ with respect to $\mathbf{x}$ is denoted by $K(\mathbf{x})$. For an $R$-module $M$, the $i$ th Koszul cohomology of $M$ with respect to $\mathbf{x}$, denoted by $\mathrm{H}^{i}(\mathbf{x} ; M)$, is the $i$ th cohomology of $\operatorname{Hom}_{R}(K(\mathbf{x}), M)$. For $1 \leq s \leq t$ there exist canonical chain maps $\phi_{s}^{t}: K\left(\mathbf{x}^{t}\right) \rightarrow K\left(\mathbf{x}^{s}\right)$ which induce natural homomorphisms

$$
\mathrm{H}^{i}\left(\mathbf{x}^{s} ; M\right) \rightarrow \mathrm{H}^{i}\left(\mathbf{x}^{t} ; M\right)
$$

for all $i$. By $\left[\mathrm{Gr}\right.$, Theorem 2.8], we have $\underset{\lim }{\longrightarrow} \mathrm{H}^{i}\left(\mathbf{x}^{n} ; M\right) \cong \mathrm{H}_{(\mathbf{x})}^{i}(M)$, the $i$ th local cohomology of $M$ with support in (x).

Following $[\mathrm{Hu}$, we define the limit closure of a sequence $\mathbf{x}$ on a module $M$ as follows:

Definition 2.1. Let $\mathbf{x}=x_{1}, \ldots, x_{r} \in R$ and let $M$ be an $R$-module. Define

$$
\{\mathbf{x}\}_{M}^{\lim _{M}}:=\bigcup_{n \geq 0}\left(\left(x_{1}^{n+1}, \ldots, x_{r}^{n+1}\right) M:_{M} x_{1}^{n} \cdots x_{r}^{n}\right) .
$$

Remark 2.2. For a sequence $\mathbf{x}=x_{1}, \ldots, x_{r}$ of $R$ consider the direct system $\left\{M /\left(\mathbf{x}^{n}\right) M\right\}_{n \geq 1}$ given by the maps

$$
M /\left(\mathbf{x}^{s}\right) M \stackrel{\left(x_{1} \cdots x_{r}\right)^{t-s}}{\longrightarrow} M /\left(\mathbf{x}^{t}\right) M
$$

for $1 \leq s \leq t$. Then the kernel of the canonical map

$$
M /(\mathbf{x}) M \rightarrow \underset{\lim }{\longrightarrow} M /\left(\mathbf{x}^{n}\right) M \cong \mathrm{H}_{(\mathbf{x})}^{r}(M)
$$


is $\left\{x_{1}, \ldots, x_{r}\right\}_{M}^{\lim } /\left(x_{1}, \ldots, x_{r}\right) M$.

The following proposition is known in a more general setting [St, Theorem 5.2.3], but we include a brief proof for the reader's convenience.

Proposition 2.3. Let $x_{1}, \ldots, x_{r} \in m$ and let $M$ be a finitely generated $R$-module. Assume that $\left\{x_{1}, \ldots, x_{r}\right\}_{M}^{\lim }=\left(x_{1}, \ldots, x_{r}\right) M$. Then $x_{1}, \ldots, x_{r}$ is a regular sequence on $M$.

Proof. We proceed by induction on $r$. In the case $r=1$, let $x=x_{1}$. Suppose $\{x\}_{M}^{\lim }=(x) M$ and $x \alpha=0$ for some $\alpha \in M$. We claim that $\alpha \in\left(x^{k}\right) M$ for all $k \geq 0$. This is clearly true for $k=0$, so suppose $\alpha=x^{k} \beta$ for some $k \geq 0$ and $\beta \in M$. Then $x^{k+1} \beta=0$, and thus $\beta \in\{x\}_{M}^{\lim }=(x) M$. Hence, $\alpha \in\left(x^{k+1}\right) M$. As $x$ is in the Jacobson radical and $M$ is finitely generated, $\bigcap_{k}\left(x^{k}\right) M=0$ by Krull's Intersection Theorem. Hence, $\alpha=0$ and $x$ is a non-zero-divisor on $M$.

Suppose now that $r>1$. To complete the proof, we will show the following:

(1) $\left\{x_{1}, \ldots, x_{r-1}\right\}_{M}^{\lim }=\left(x_{1}, \ldots, x_{r-1}\right) M$.

(2) $x_{r}$ is a non-zero-divisor on $M /\left(x_{1}, \ldots, x_{r-1}\right) M$.

Item (1) will allow us to use the inductive hypothesis to conclude that $x_{1}, \ldots, x_{r-1}$ is a regular sequence on $M$.

To prove (1), let $\alpha \in\left\{x_{1}, \ldots, x_{r-1}\right\}_{M}^{\lim }$. We claim that for all $k \geq 0, \alpha \in$ $\left(x_{1}, \ldots, x_{r-1}\right) M+\left(x_{r}^{k}\right) M$. Again by Krull's Intersection Theorem, this will imply that $\alpha \in\left(x_{1}, \ldots, x_{r-1}\right) M$. The case $k=0$ is clear, so suppose $\alpha=\omega+x_{r}^{k} \beta$ where $\omega \in\left(x_{1}, \ldots, x_{r-1}\right) M$ and $\beta \in M$. Thus, $x_{r}^{k} \beta \in\left\{x_{1}, \ldots, x_{r-1}\right\}_{M}^{\lim }$. Hence, there exists $t \geq 0$ such that

$$
\left(x_{1} \cdots x_{r-1}\right)^{t} x_{r}^{k} \beta \in\left(x_{1}^{t+1}, \ldots, x_{r-1}^{t+1}\right) M .
$$

Multiplying by $\left(x_{1} \cdots x_{r-1}\right)^{k} x_{r}^{t}$, we obtain

$$
\left(x_{1} \cdots x_{r}\right)^{t+k} \beta \in\left(x_{1}^{t+k+1}, \ldots, x_{r-1}^{t+k+1}\right) M \subseteq\left(x_{1}^{t+k+1}, \ldots, x_{r}^{t+k+1}\right) M .
$$

Hence, $\beta \in\left\{x_{1}, \ldots, x_{r}\right\}_{M}^{\lim }=\left(x_{1}, \ldots, x_{r}\right) M$. Thus, $\alpha \in\left(x_{1}, \ldots, x_{r-1}\right) M+\left(x_{r}^{k+1}\right) M$.

The proof of (2) is similar: Suppose $x_{r} \alpha \in\left(x_{1}, \ldots, x_{r-1}\right) M$ for some $\alpha \in M$. We claim that $\alpha \in\left(x_{1}, \ldots, x_{r-1}\right) M+\left(x_{r}^{k}\right) M$ for all $k \geq 0$. Suppose $\alpha=\omega+x_{r}^{k} \beta$ where $\omega \in\left(x_{1}, \ldots, x_{r-1}\right) M$ and $\beta \in M$. Then $x_{r} \alpha=x_{r} \omega+x_{r}^{k+1} \beta$. Hence, $x_{r}^{k+1} \beta \in\left(x_{1}, \ldots, x_{r-1}\right) M$. Multiplying by $\left(x_{1} \cdots x_{r-1}\right)^{k+1}$, we obtain that

$$
\left(x_{1} \cdots x_{r}\right)^{k+1} \beta \in\left(x_{1}^{k+2}, \ldots, x_{r-1}^{k+2}\right) M \subseteq\left(x_{1}^{k+2}, \ldots, x_{r}^{k+2}\right) M .
$$

Hence, $\beta \in\left\{x_{1}, \ldots, x_{r}\right\}_{M}^{\lim }=\left(x_{1}, \ldots, x_{r}\right) M$ and $\alpha \in\left(x_{1}, \ldots, x_{r-1}\right) M+\left(x_{r}^{k+1}\right) M$.

Definition 2.4. Let $(R, m)$ be a local ring, let $M$ be a finitely generated $R$-module, and let $i \geq 0$. We will define the invariant $\ell_{i}(M)$. By applying $\operatorname{Ext}_{R}^{i}(-, M)$ to the system of surjections

$$
\cdots \rightarrow R / m^{3} \rightarrow R / m^{2} \rightarrow R / m
$$

we obtain a direct system whose limit is $\lim _{\longrightarrow} \operatorname{Ext}_{R}^{i}\left(R / m^{n}, M\right) \cong \mathrm{H}_{m}^{i}(M)$. Since direct limits commute with $\operatorname{Hom}_{R}(R / m,-)$,

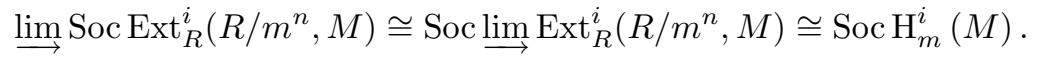


Since $\mathrm{H}_{m}^{i}(M)$ is Artinian, Soc $\mathrm{H}_{m}^{i}(M)$ is finitely generated, and so there exists a

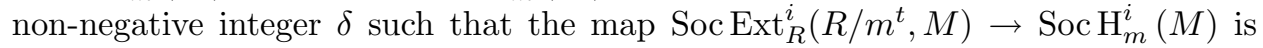
surjective for all $t \geq \delta$. We define $\ell_{i}(M)$ to be the least integer $\delta$ with this property.

Let $M$ be a finitely generated $R$-module. We say an ideal $q=\left(x_{1}, \ldots, x_{d}\right)$ is a parameter ideal for $M$ if $d=\operatorname{dim} M$ and $\operatorname{dim} M / q M=0$. The following proposition is essentially [GSa1, Lemma 3.12].

Proposition 2.5 (GSa1, Lemma 3.12]). Let $(R, m)$ be a Noetherian local ring, let $M$ be a finitely generated $R$-module of dimension $d$, and let $i \geq 0$. For all systems $x_{1}, \ldots, x_{d}=\mathbf{x}$ of parameters for $M$ contained in $m^{\ell_{i}(M)}$, the map

$$
\mathrm{Soc}^{i}(\mathbf{x} ; M) \rightarrow \operatorname{Soc~}_{m}^{i}(M)
$$

induced by the canonical map $\mathrm{H}^{i}(\mathbf{x} ; M) \rightarrow \underline{\lim } \mathrm{H}^{i}\left(\mathbf{x}^{n} ; M\right)$ is surjective.

Remark 2.6. There are two differences between the above result and GSa1, Lemma 3.12]. The first difference is that in [GSa1, Lemma 3.12], instead of defining each integer $\ell_{i}(M)$ independently, the integer $\max _{i=1}^{d} \ell_{i}(M)$ is used so that one power of the maximal ideal works to make all of the canonical maps surjective on the socles. In this paper, we only need Proposition 2.5 for the $d$ th local cohomology module.

The second difference is that the result [GSa1, Lemma 3.12] is stated for systems of parameters of the ring $R$ instead of the module $M$. However, this is remedied in GSa2, Corollary 2.15] by passing to the $\operatorname{ring} R / \operatorname{Ann}_{R} M$.

The main result now follows readily from the two propositions above:

Theorem 2.7. Let $(R, m)$ be a Noetherian local ring, let $M$ be a finitely generated $R$-module of dimension $d$, and let $\ell=\ell_{d}(M)$. Then $M$ is Cohen-Macaulay with Cohen-Macaulay type $r(M)=1$ if and only if some parameter ideal $q$ for $M$ contained in $m^{\ell}$ has the property that $q M$ is irreducible.

Proof. It suffices to show that if there exists a system of parameters $\mathbf{x}=x_{1}, \ldots, x_{d}$ for $M$ contained in $m^{\ell}$ such that $(\mathbf{x}) M$ is irreducible, then $M$ is Cohen-Macaulay. Let $\phi$ denote the canonical homomorphism from $H^{d}(\mathbf{x} ; M) \cong M /(\mathbf{x}) M$ to

$$
\lim _{\longrightarrow} H^{d}\left(\mathbf{x}^{n} ; M\right) \cong \mathrm{H}_{m}^{d}(M) \text {. }
$$

By Remark 2.2 we have an exact sequence

$$
0 \longrightarrow \frac{\{\mathbf{x}\}_{M}^{\lim }}{(\mathbf{x}) M} \longrightarrow \frac{M}{(\mathbf{x}) M} \stackrel{\phi}{\longrightarrow} \mathrm{H}_{m}^{d}(M)
$$

Applying the socle functor and using Proposition 2.5 we obtain the exact sequence

$$
0 \longrightarrow \operatorname{Soc} \frac{\{\mathbf{x}\}_{M}^{\lim }}{(\mathbf{x}) M} \longrightarrow \operatorname{Soc} \frac{M}{(\mathbf{x}) M} \longrightarrow \operatorname{Soc}_{m}^{d}(M) \longrightarrow 0
$$

Since $\mathrm{H}_{m}^{d}(M)$ is a non-zero Artinian module, it has a non-zero socle. Since $(\mathbf{x}) M$ is irreducible, $M /(\mathbf{x}) M$ has a one-dimensional socle. Hence, $\operatorname{Soc}\left(\{\mathbf{x}\}_{M}^{\lim } /(\mathbf{x}) M\right)=0$, which implies $\{\mathbf{x}\}_{M}^{\lim }=(\mathbf{x}) M$. By Proposition 2.3, we see that $\mathbf{x}$ is $M$-regular, $M$ is Cohen-Macaulay and $r(M)=\operatorname{dim}_{R / m} \operatorname{Soc} H_{m}^{d}(M)=1$.

Corollary 2.8. Let $(R, m)$ be a Noetherian local ring. Then $R$ is Gorenstein if and only if every power of the maximal ideal contains an irreducible parameter ideal.

Proof. Take $M=R$ in Theorem 2.7 . 


\section{ACKNOWLEDGMENTS}

The authors would like to thank Shiro Goto, William Heinzer, Craig Huneke and Jung-Chen Liu for many helpful comments during the preparation of this article.

\section{REFERENCES}

[BH] Bruns, W. and Herzog, J., Cohen-Macaulay Rings, Cambridge Studies in Advanced Mathematics no. 39, Cambridge, Cambridge University Press, 1993. MR.1251956 (95h:13020)

[G] Goto, S., Approximately Cohen-Macaulay rings, J. Algebra 76 (1982), 214-225. MR659220 (84h:13033)

[GSa1] Goto, S. and Sakurai, H., The equality $I^{2}=Q I$ in Buchsbaum rings, Rend. Sem. Mat. Univ. Padova 110 (2003), 25-56. MR2033000 (2004m:13061)

[GSa2] Goto, S. and Sakurai, H., Index of Reducibility of Parameter Ideals for Modules Possessing Finite Local Cohomology Modules, Preprint.

[Gr] Grothendieck, A., Local Cohomology, notes by R. Hartshorne, Lect. Notes Math. no. 41, Springer, Berlin, 1966. MR0224620 (37:219)

[Ho] Hochster, M., Cyclic purity versus purity in excellent Noetherian rings, Trans. Am. Math. Soc. 231 (1977), no. 2, 463-488. MR0463152 (57:3111)

[Hu] Huneke, C., Tight closure, parameter ideals, and geometry, in Six Lectures on Commutative Algebra (J. Elias, J.M. Giral, R.M. Miró-Roig, and S. Zarzuela, eds.), Progress in Mathematics, vol. 166, Birkhäuser Verlag, Basel, 1998, 187-239. MR1648666 (99j:13001)

[LR] Liu, J. C. and Rogers, M., The index of reducibility of parameter ideals and mostly zero finite local cohomologies, Comm. Algebra, to appear.

[Mat] Matsumura, H., Commutative Ring Theory, Cambridge Studies in Advanced Mathematics no. 8, Cambridge, Cambridge University Press, 1986. MR.879273 (88h:13001)

[NR] Northcott, D. G. and Rees, D., Principal Systems, Quart. J. Math. 8 (1957), 119-127. MR.0096649(20:3132)

[R] Rogers, M., The index of reducibility for parameter ideals in low dimension, J. Alg., 278/2 (2004), 571-584. MR2071653 (2005e:13026)

[St] Strooker, J. R., Homological questions in local algebra, London Math. Soc. Lecture Note series 145, Cambridge Univ. Press, Cambridge 1990. MR.1074178 (91m:13013)

Department of Mathematics, University of Nebraska, Lincoln, Nebraska 68588-0130

E-mail address: tmarley@math.unl.edu

Department of Mathematics, Missouri State University, Springfield, Missouri 65897

E-mail address: markrogers@missouristate.edu

Department of Mathematics, School of Science and Technology, Meiji University, 214-8571, JAPAN

E-mail address: hsakurai@math.meiji.ac.jp 